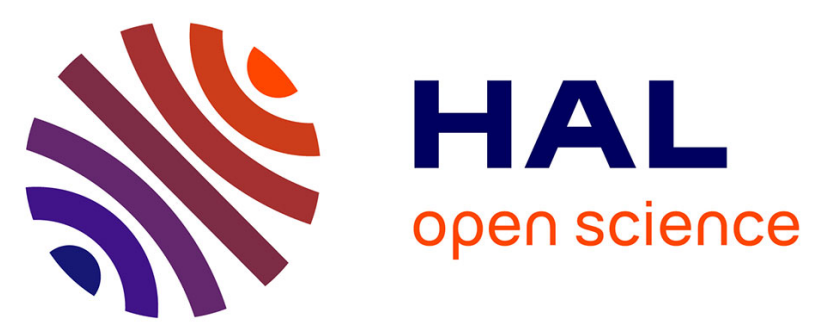

\title{
Vocalizing Dance Movement for Interactive Sonification of Laban Effort Factors
}

Jules Françoise, Sarah Fdili Alaoui, Thecla Schiphorst, Frédéric Bevilacqua

\section{To cite this version:}

Jules Françoise, Sarah Fdili Alaoui, Thecla Schiphorst, Frédéric Bevilacqua. Vocalizing Dance Movement for Interactive Sonification of Laban Effort Factors. Proceedings of the 2014 Conference on Designing Interactive Systems, Jun 2014, Vancouver, Canada, France. pp.1079-1082, $10.1145 / 2598510.2598582$. hal-01061224

\section{HAL Id: hal-01061224 \\ https://hal.science/hal-01061224}

Submitted on 5 Sep 2014

HAL is a multi-disciplinary open access archive for the deposit and dissemination of scientific research documents, whether they are published or not. The documents may come from teaching and research institutions in France or abroad, or from public or private research centers.
L'archive ouverte pluridisciplinaire $\mathbf{H A L}$, est destinée au dépôt et à la diffusion de documents scientifiques de niveau recherche, publiés ou non, émanant des établissements d'enseignement et de recherche français ou étrangers, des laboratoires publics ou privés. 


\title{
Vocalizing Dance Movement for Interactive Sonification of Laban Effort Factors
}

\author{
Jules Françoise*, Sarah Fdili Alaoui ${ }^{\dagger}$, Thecla Schiphorst ${ }^{\dagger}$, Frédéric Bevilacqua* \\ * STMS Lab, IRCAM-CNRS-UPMC, Paris, France. \\ $\dagger$ School of Interactive Arts and Technologies, Simon Fraser University, Surrey, British Columbia, \\ Canada. \\ jules.francoise@ircam.fr, frederic.bevilacqua@ircam.fr, sfdilial@sfu.ca, thecla@sfu.ca
}

\begin{abstract}
We investigate the use of interactive sound feedback for dance pedagogy based on the practice of vocalizing while moving. Our goal is to allow dancers to access a greater range of expressive movement qualities through vocalization. We propose a methodology for the sonification of Effort Factors, as defined in Laban Movement Analysis, based on vocalizations performed by movement experts. Based on the experiential outcomes of an exploratory workshop, we propose a set of design guidelines that can be applied to interactive sonification systems for learning to perform Laban Effort Factors in a dance pedagogy context.
\end{abstract}

\section{Author Keywords}

Dance; Laban Movement Analysis; Effort Factors, Movement Qualities; Interactive Sonification; Vocalization; Motion-Sound Mapping; Multimodal.

\section{ACM Classification Keywords}

H.5.5. Information Interfaces and Presentation (e.g. HCI): Sound and Music Computing; J.5. Computer Applications: Arts and Humanities

\section{INTRODUCTION}

We naturally interact with our environment using voice and gesture, and not surprisingly voice and gesture are, two major input modalities utilized in Human Computer Interaction (HCI). Historically, gesture control and speech recognition have been utilized for various discrete functional tasks such as triggering actions. But with the advent of HCI's "third wave" [3], there is a shift of interest towards interaction modalities that incorporate elements such as expressivity, meaning, engagement and emotion.

The work that we report here is part of an ambitious research agenda focusing on movement expressivity in HCI through the use of movement qualities as an interaction modality.

Permission to make digital or hard copies of all or part of this work for personal or classroom use is granted without fee provided that copies are not made or distributed for profit or commercial advantage and that copies bear this notice and the full citation on the first page. Copyrights for components of this work owned by others than the author(s) must be honored. Abstracting with credit is permitted. To copy otherwise, or republish, to post on servers or to redistribute to lists, requires prior specific permission and/or a fee. Request permissions from Permissions@acm.org. DIS'14, June 21 - 25 2014, Vancouver, BC, Canada.

Copyright is held by the owner/author(s). Publication rights licensed to ACM ACM 978-1-4503-2902-6/14/06 \$15.00.

http://dx.doi.org/10.1145/2598510.2598582
Fdili Alaoui et al. [5] describe movement qualities as a qualitative attribute of moment produced by dynamics and defining the ways movement is executed. In this paper, we report on a specific aspect of this research: investigating the use of interactive sound feedback to reflect and guide the performance of movement qualities as defined in the Effort category of the Laban Movement Analysis (LMA) framework. LMA formalizes movement qualities as Effort, a category that describes how a movement is performed with respect to the mover's inner attitude or intention.

We propose a methodology for the interactive sonification of Effort Factors that draws from vocalizations performed by Certified Laban Movement Analysts (CMAs). Our interactive system is built upon machine learning methods that learn the mapping between movement and sound from expert performances. We evaluated our movement-sound interaction models and the associated sonification systems in a workshop where dancers were taught to perform and experience Laban's Effort Factors. The workshop used bodystorming and focus group open-ended interviewing techniques to elicit participant feedback regarding design, technological, and experiential issues of voice-based sonic interactions in dance pedagogy. This format allowed us to assess the participants' experience of the interactive sonic feedback and to establish guidelines for further development of sonic systems dedicated to movement qualities.

\section{RELATED WORK}

Few interactive systems exploit movement qualities as an interaction modality, particularly for dance or artistic installations, and they rarely include interactive sonic feedback to support movement expression. Camurri et al. developed a framework for music and dance applications to analyze and classify expressive gestures along Laban Effort Factors using the Eyesweb platform [2]. Schiphorst used Laban's Effort Factors to enhance body awareness and the æsthetic experience of interacting with tangible digital media [10]. Fdili Alaoui et al. have recently shown that movement qualities can enhance user experience and exploration in interactive installations [5], and such a system was evaluated and used in dance pedagogy [4]. Maranan et al. modeled Efforts using a single-accelerometer system, for interactive visualizations in dance performances and installation [8]. Mentis and Johanson proposed a study that aims to situate the perception of Effort Factors, through a Kinect-based system for an improvisational dance installation in which the recognition of 
audience members' Effort Factors trigger musical events [9]. However, to the best of our knowledge, no system addresses the sonification of Laban's Effort Factors for dance pedagogical purposes.

\section{EFFORT IN LABAN MOVEMENT ANALYSIS}

LMA is a system that provides rigorous models for the description of movement, its function and its expression through four components, defined as Body, Effort, Space and Shape. In this paper, we investigate the qualitative aspects of movement that conveys movement expressiveness, as defined in the Effort category of LMA. Effort can be experienced and observed as an attitude shift that reveals the mover's intent in response to the environment [7]. It encompasses 4 discrete Factors: Space, Time, Weight, and Flow. Each Effort Factor is thought of as a continuum between two opposite 'Factors' in which movement can vary and thus reveal different 'qualities' [7]. Space is related to one's attention to the surrounding environment either through scanning the whole environment (Indirect Space) or focusing on a single element (Direct Space). Time is related to one's inner attitude to time expressed either through acceleration (Quick Time) or deceleration (Sustained Time). Weight is related to one's resistance to gravity through increasing (Strong Weight) or decreasing pressure (Light Weight) using the muscular tension. Flow is the experience and expression of the ongoingness of movement that determines how movement is released (Free Flow) or controlled (Bound Flow).

\section{Breath Support in LMA}

Breath is the first form of human movement, and breath continues to support the development and expression of all human movement throughout our life cycle. According to Irmagard Bartenieff, one of the major historical figures of LMA, "Movement rides on the flow of Breath" [1]. The use of Breath in LMA allows the human body to access a large palette of movements, by supporting the phrasing of movement and the full body shaping. Bartenieff emphasizes the crucial role of vocalization as an amplification of Breath in achieving a fluidity and expressivity in movement

\section{MOVEMENT SONIFICATION BASED ON VOCALIZATION}

Our approach to movement sonification uses vocalizations performed by CMAs along with movement with various Effort Factors. We have developed a sonification system based on a method called Mapping-by-Demonstration [6]. Mapping refers to the relationship between the movement features and the sound parameters, which design is central to any movement-sound interactive system. Rather than formulating an analytical description of the relationship between movement and sound, Mapping-by-Demonstration trains a machine learning model on examples of movements associated with sounds in order to learn the motion-sound interaction model. Technically, our system is based on a set of Multimodal Hidden Markov Models (MHMMs) that provide a multimodal representation of movements and sounds, thus capturing the temporal dynamics and expressive variations of the movement-sound relationship [6].
The workflow of the system is shown in Figure 1. First, during the Training phrase, we synchronously record CMAs' vocalizations and movements to form a set of multimodal sequences of movement features extracted from sensors data, and voice features computed from audio. From the set of associated movements and vocalizations, the system learns the relationship between movement features and voice features. The system is then used for interactive sonification: during the Performance phase, the participants' live movements are interpreted by the system that continuously estimates the associated voice features in order to re-synthesize the pre-recorded vocalizations using corpus-based sound synthesis [11].

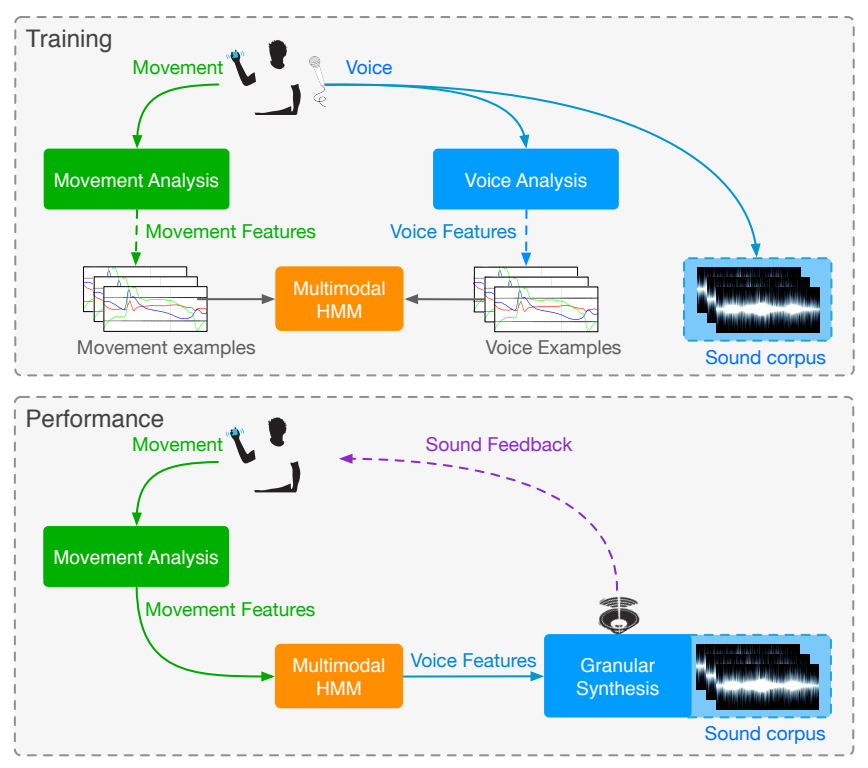

Figure 1. Overview of the vocalization system.

We recorded two CMAs, Karen Studd and Philippe Seth, vocalizing and performing movements with Sustained or Quick Time Effort or Strong or Light Weight Effort. Each CMA was asked to vocalize throughout her movement performance using breath support with the intent of producing a sound quality that subjectively 'represented' the performed Effort. Each Vocalization was recorded synchronously with multimodal sensor data. Previous experiments allowed us to derive a set of sensors and movement features that are useful to characterize Effort Factors through the formalization of expert CMAs' observational process. In this study we chose to focus on Time and Weight Effort Factors, because they are the most accurately recognized in real-time using the proposed sensors and features.

We used two different types of sensors to record the movement data: a 3D accelerometer attached to the right wrist and an electromyography sensor (EMG) attached to the forearm (see Figure 2). Data was sampled at $200 \mathrm{~Hz}$ and transmitted wirelessly to a computer. A microphone, connected to the same computer, was used to record the CMA's vocalization. The movement features selected to represent Time and Weight Effort Factor are respectively: 
Time Effort Factor: magnitude of the derivative of the acceleration measured with a 3D accelerometer placed on the right wrist. This feature correlates with the sense of acceleration and deceleration that CMAs use to observe Quick versus Sustained Time Effort.

Weight Effort Factor: intensity of the muscular activation evaluated using a non-linear Bayesian filtering of Electromyography (EMG), captured with an EMG sensor placed on the right forearm. The muscular tension variation correlate to the experience of Strong versus Light Weight Effort.

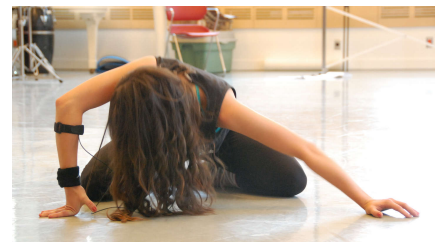

Figure 2. A participant equipped with the sensors: 3D accelerometer (wrist) and EMG (forearm).

We developed two separate models for Time or Weight Effort Factor. Each model was trained using 24 pre-recorded vocalizations and performance of movements with Sustained or Quick Time Efforts or Strong or Light Weight Efforts. These models are then used for interactive sonification: a dancer equipped with sensors (accelerometers and EMG) can control the re-synthesis of the CMAs' vocalizations (either Time or Weight Effort Factors).

\section{EVALUATION WORKSHOP}

We organized a one-day workshop with dance participants that learned Laban Efforts through interactive sonification. The workshop was facilitated by CMA Donna Redlick, and the dancers were observed by CMAs Michelle Olson and Leslie Bishko. The facilitator was asked to use the interactive sonification system to support the teaching of Effort Factors.

In the first session, the participants were given an overview of LMA and began to explore its structure through experiential sessions led by Donna Redlick. In the second session, participants were equipped with the set of sensors, and interacted with the sonic system. During both sessions they were guided by the facilitator, and their performance of the Effort Factors was observed and analyzed by two other CMAs. Observers and participants were encouraged to talk aloud about their experience or observations using their own terminology and including, when possible, LMA terminology. During the interactive session, each participant was guided by the facilitator to improvise with the sonic feedback in order to experience the targeted Effort factor and exhibit qualities at the extreme end of the Time and Weight Factors. Later, other participants could join and experience the targeted Effort Factor by attuning either to the movement of the equipped participant or to the produced sound.

\section{Participants}

We recruited 5 participants (all female between 20 and 40 years old), with several years of dance practice, but no prior knowledge or practice of LMA. Participants were all comfortable with improvising and discussing their experience of movement exploration as well as being equipped with sensors and interacting with the sonic feedback.

\section{Data Analysis}

The workshop was videotaped and the observations of the three CMAs were recorded. We used qualitative analysis for the transcriptions of the observations and discussions within the group in order to assess the participants' experience of the interactive sonic feedback and to capture the emerging guidelines for the design of sonic systems dedicated to movement training. We specifically focused on the comments relating to the effect of the interactive sound feedback on the participants' movement; the relationship between the movement, the sound, and the observed Effort Factors; and the experience of the interaction itself.

\section{RESULTS}

We report here the main results that emerge from the qualitative analysis of open discussions among the group. We refer to participants as P1, P2, etc.; to observers as CMA1, CMA2; and to the facilitator as F. We use the terms 'mover' to designate the participant in interaction. All citations come from transcription.

\section{Movement to Sound}

Open discussions brought out several issues about the effect of the interactive sonification on participants' movements, highlighting strengths and limitations in the design of the movement-sound relationship.

The sonification of the Weight Effort Factor was considered as responsive and consistent. Particular sounds were often revealed through specific movement patterns embodying the Weight Effort Factor: "Now she [P2] is playing with that weight sense, and that contact [to the floor] gets the sound". Absence of Weight Effort Factor was also revealed through the sound, thus allowing to better access Weight Effort Factor: "She went to vision drive and there was no sound. Vision Drive is weightless. It is Space, Time And Flow. And it was interesting because the sound stopped." $(F){ }^{1}$

Time Effort Factor sonification suffered from latency due to a filtering of the results intended to improve the recognition and smooth the sound feedback. Moreover, the relationship between Effort and sound was not perceived as transparent for Time Effort sonification. Several participants commented that the feedback contained much more information than Time only, often correlating it to Weight. These comments suggest the difficulty, highlighted by the CMAs during the recording sessions, to perform and vocalize Time Effort as an isolated Factor, and in that case to design movement-sound mappings.

Finally, inter-subject variability was brought up by observers who noticed different sound outcomes according to the mover's personal palette. Indeed some participants naturally accessed Time or Weight nuances more easily in their movement signature: "I was hearing more sounds in this palette

${ }^{1}$ LMA defined four Effort Drives combining three Effort factors and missing one Effort factor. Action Drives miss Flow; Spell Drives miss Time, Vision Drives miss Weight, and Passion Drives miss Space Effort. 
that I didn't hear in other people's movements." (CMA2). This observation might correlate to the issue of sensor calibration, yet it also points to the nature of differing movement signatures elicited by each human body. In particular, while muscular activation highly varies from one participant to another, it also requires fine-tuning for each participant.

\section{Sound to Movement}

Movers consistently reported an experience of attuning to the sound, often engaging in an exploration of movement directed towards the production of sound: "I was trying to figure out how to make a sound, knowing that my body had the vocabulary to do it." (P1). Besides, wearing the sensing devices themselves seemed to influence the participants' behavior, as reported by CMA2 who noticed a "more isolated use of body parts" of the equipped participant. In such cases the facilitator guided the movers towards an exploration of other body parts, often resulting in changes in the sound feedback: "You were making some very new and interesting sound when initiating from the torso." (P2). Finally, the sound feedback influenced the performance of Effort Factors. CMA2 reported on a portion of the interactive session during which all participants were improvising with Time Effort Factor: "There was often very percussive sounds that I think were stimulating everyone to go into Quick Effort.". Due to the ambiguities of Time Effort Factor sonification, the feedback could sometimes lead to changes in Effort that didn't relate to the sonified Effort Factor: "what I didn't see in you moving before [before interacting with sound] is you increasing pressure. Adding weight to your vocabulary." (F).

The CMAs and participants unanimously acknowledged the potential for new understanding, support for pedagogical opportunities afforded by technology, and the creation of a reflective space for learning.

\section{DISCUSSION AND CONCLUSION}

We have reported the results of a workshop intended to evaluate an approach to the sonification of Laban Effort Factors based on experts' vocalizations. The participants and experts had a very positive experience of the workshop and acknowledged its potential for supporting a better understanding of Effort Factors particularly within dance pedagogy. Several guidelines emerge from the discussions between participants and experts, providing precious insights for future development of such interactive sonic systems. First, the results stress the importance of tightening the relationship between movement and sound by limiting the latency and guaranteeing the transparency of the mapping between Effort Factors and sound. Technically, this requires a thorough selection of the training examples with a specific focus on quality, consistency, and alignment. In particular, the intrinsic difficulty of articulating the vocalization and performance of isolated Effort Factors argues for the need of in-depth studies that correlate vocalizations' perceptive attributes with the identification of movement qualities. Finally, the very personal nature of Effort performance and experience questions the transferability of the Effort models among movers. This aspect motivates the development of higher-level movement features and richer computational models of Effort Factors that can adapt to a mover's personal signature by continuously learning during interaction.

\section{ACKNOWLEDGMENTS}

We acknowledge support from the MovingStories project (http://movingstories.ca/) and from the ANR project Legos 11 BS02 012. We thank CMAs Donna Redlick, Michelle Olson, Leslie Bishko, Philip Seth, and Karen Studd for their involvement in the workshop or the Motion Capture session.

\section{REFERENCES}

1. Bartenieff, I. Body movement: Coping with the environment. Routledge, 1980.

2. Camurri, A., Hashimoto, S., Ricchetti, M., Ricci, A., Suzuki, K., Trocca, R., and Volpe, G. Eyesweb: Toward gesture and affect recognition in interactive dance and music systems. Computer Music Journal volume 24, 1 (2000), pages 57-69.

3. Fallman, D. The new good: exploring the potential of philosophy of technology to contribute to human-computer interaction. In Proceedings of the SIGCHI Conference on Human Factors in Computing Systems, ACM (2011), 1051-1060.

4. Fdili Alaoui, S., Bevilacqua, F., Pascual, B. B., and Jacquemin, C. Dance interaction with physical model visuals based on movement qualities. International Journal of Arts and Technology 6, 4 (2013), 357-387.

5. Fdili Alaoui, S., Caramiaux, B., Serrano, M., and Bevilacqua, F. Movement Qualities as Interaction Modality. In ACM Designing Interactive Systems (DIS'12) (Newcastle, UK, 2012).

6. Françoise, J., Schnell, N., and Bevilacqua, F. A Multimodal Probabilistic Model for Gesture-based Control of Sound Synthesis. In Proceedings of the 21st ACM international conference on Multimedia (MM'13) (Barcelona, Spain, 2013), 705-708.

7. Laban, R., and Lawrence, F. C. Effort. London: MacDonald and Evans., 1947.

8. Maranan, D. S., Fdili Alaoui, S., Schiphorst, T., Pasquier, P., and Bartram, L. Designing For Movement: Evaluating Computational Models using LMA Effort Qualities. In Proceedings of the SIGCHI Conference on Human Factors in Computing Systems (2014).

9. Mentis, H. M., and Johansson, C. Seeing movement qualities. In Proceedings of the SIGCHI Conference on Human Factors in Computing Systems (2013), 3375-3384.

10. Schiphorst, T. soft (n): Toward a Somaesthetics of Touch. In Proceedings of the SIGCHI Conference on Human Factors in Computing Systems (2009), 2427-2438.

11. Schwarz, D. Corpus-based concatenative synthesis. Signal Processing Magazine, IEEE 24, 2 (2007), 92-104. 